\title{
Statistical Analysis of Elements of Movement in Musical Expression in Early Childhood Using 3D Motion Capture and Evaluation of Musical Development Degrees Through Machine Learning
}

\author{
Mina Sano ${ }^{1, *}$ \\ ${ }^{1}$ Department of Childhood Education, Osaka-Shoin-Women's University, Higashiosaka, Japan \\ *Correspondence: Mina Sano, Department of Childhood Education, Osaka-Shoin-Women's University, \\ Higashiosaka, Japan. Tel: 816-7507-1472. E-mail: minasano@yahoo.co.jp
}

Received: May 25, 2018

Accepted: June 21, $2018 \quad$ Online Published: June 25, 2018

doi:10.5430/wje.v8n3p118

URL: https://doi.org/10.5430/wje.v8n3p118

\begin{abstract}
This study aims to analyze the developmental characteristics of early childhood musical expressions from a viewpoint of movement elements, and to devise a method to evaluate the development regarding musical expression in early childhood using machine learning. Previous studies regarding motion capture have shown analysis results such as specific actions and responses to music (Burger et al, 2013). In this study, firstly, ANOVA was attempted on full-body movements. The author quantitatively analyzed the motion capture data regarding 3-year-old, 4-year-old, and 5-year-old children in the nursery schools $(n=84)$ and kindergartens $(n=94)$ through a three-way non-repeated ANOVA. As a result, a statistically significant difference was observed in movement of body parts. Specifically, right hand movement such as moving distance and the moving average acceleration showed a significance of difference. Secondly, machine learning (decision trees, Sequential Minimum Optimization algorithm (SMO), Support Vector Machine (SVM) and neural network (multilayer perceptron)) was deployed to build classification models for evaluation of degree of musical development classified by educators with simultaneously recorded children's video with associated motion capture data. Among varieties of trained classification models, multilayer perceptron obtained best results of confusion matrix and showed fair classifying precision and usability to support educators to evaluate children's achievement degree of musical development. As a result of the machine learning of multilayered perceptron, the movement of the pelvis has a strong relationship with musical development degree. Its classification accuracy found consistent to affirm the availability to utilize the model to support educators to evaluate children's attainment of musical expression.
\end{abstract}

Keywords: 3D motion capture, musical expression in early childhood, ANOVA, machine learning, multilayer perceptron

\section{Introduction}

Early childhood children spontaneously pretend and dramatize in everyday play and musical expression and express them using their whole body. It is inevitable for children to express spontaneously body movement in musical expression during early childhood. Early childhood children often create the movement of pretend while singing and replay a story during singing play. The author devised the MEB (Musical Expression Bringing-up) program which made use of such developmental characteristics in early childhood referred to several previous studies which suggested the importance of movement in musical expression to integrate dramatization with music education (Winston \& Tandy, 2005; Ballema, 2005; Rubin \& Merrion, 1996). The MEB program consists of four phase's activity such as (1) beginning activity, (2) pantomime and improvisation, (3) story creation, and (4) dramatization of the story. MEB program has a main purpose to encourage the recognition of musical elements in early childhood. The program begins to establish the image of a phenomenon of everyday life as the activity, such as a name game, song play, or sound awareness and advance to the formation of a rhythmic pattern and reply song with body movement such as dramatization. 3-year-old, 4-year-old, and 5-year-old children in nursery school and kindergarten participated in the musical expression program, and the educational effect was qualitatively analyzed (Sano, 2015).

The author also devised the music test constituted of 6 domains such as"Strength of sound," "Duration of sound," 
"Rhythm," "Pitch of sound," "Harmony of sound", and "Expression and appreciation" and every domains include 10 contents (Sano, 2014). The music test before and after practice of MEB program was applied to 4-year-old and 5-year-old children so that the educational effect was quantitatively analyzed using ANOVA and PCA (Sano, 2013). In previous studies, mainly experimental results were showed about the recognition of rhythm and melody by infants (Hannon \& Johnson, 2005; Phiilips- Silver \& Trainor, 2005) and children' response to sound (Zentner \& Eerola, 2010) although various case studies regarding a relationship between the movement and the recognition of musical elements were indicated (Thelen, 1979; Custodero, 1999). Those results have pointed out the importance of a relationship between the recognition of musical elements and movement.

Based on the aforementioned study results, it is considered that developmental characteristics of musical expression in early childhood can be extracted by quantitative analysis from a viewpoint of change regarding body movement in musical expression peculiar to early childhood.

Therefore, the author thought to analyze the body movement in musical expression by activity phase about the practical process of MEB program mentioned above using 3D motion capture. Previous studies which adopted 3D motion capture in the field of education showed discussion results of specific movement analysis of Japanese traditional dancing and sawing mainly for adults and development of learning support method (Sato et al. 2010). Previous studies regarding music and movement (Burger, 2013), audience experiment and video analysis on the relationship between performer's actions and expression (Dahl \& Friberg, 2007; Thompson \& Luck, 2012) did not intend analyze musical expression in early childhood.

The author firstly tried to use MTw system to wear a motion tracker one by one for each young child and at the same time to analyze the movement of five children at the same time in the practical process of musical expressions (Sano, 2016). In order to calculate the moving distance and moving trace of each young child, the author also conducted a motion analysis of a small number of children one by one using the wired connection type MVN system (Sano, 2016).

Furthermore, using the wirelessly connected MVN system, which was recently developed, compact, lightweight and easy to wear, the author have analyzed the body movement in musical expression during early childhood to acquire more various measurement data. In the MVN system, participant child was attached motion tracker to body parts of 17 places such as head, left and right shoulder, left and right upper arm, left and right lower arms, left and right hands, left and right upper limbs, left and right lower limbs, left and right legs, chest and waist, and captures the movement. Children in the two nursery schools in 2016, and children in the two kindergartens in 2017 participated in MEB program and the movement analysis about the musical expression during the practical process (Sano, 2017; Sano, 2018). The author showed a part of the results analysis such as the effects of the MEB program and characteristics of elements of movement in musical expression using a three-way, non-repeated ANOVA.

In this article, the author extracted the characteristics of musical expression from the quantitative analysis results of the elements of body movement in musical expression of the participant children in U \& K nursery schools in 2016 and F \& Y kindergartens in 2017. Nursery schools usually include children from 0-year-old to 6-year-old and kindergartens usually include children from 3-year-old to 6-year-old in Japan. From the data acquired by MVN system, the feature quantity of the element of body movement in musical expression was calculated and the author tried to carry out model classification by machine learning so that early childhood educators can judge the richer musical expression and develop a method of evaluation support.

\section{Purpose of This Study}

The purpose of this study is to extract the developmental characteristics of musical expression in early childhood from a viewpoint of change of elements of body movement and to generate an evaluation model based on those feature quantities. The author used the MVN system to acquire 3D motion capture data regarding 3-year-old, 4-year-old, and 5-year-old children in two nursery schools acquired in 2016 and two kindergartens in 2017. It is intended to evaluate the degree of development of musical expression in early childhood and constitute classification model with machine learning technology.

\section{Method}

\subsection{Practice of MEB Program and Extraction of MVN Measurement Items by Activity Phase}

Firstly, 3-year-old, 4-year-old, and 5-year-old children participated in the practice of MEB program in U \& K nursery schools $(n=120)$ in 2016 and F \& Y kindergartens $(n=194)$ in 2017. U nursery school and F kindergarten take a 
childcare form of play-centered. K nursery school and Y kindergarten take a childcare form following the Montessori Method.

Secondly, characteristic item for MVN was extracted from the MEB program by phase. MVN measurement data of four times in total, once for each activity phase was recorded.

Table 1. Activity Contents and the Measurement Dates

\begin{tabular}{|c|c|c|c|c|c|}
\hline & $\begin{array}{l}\text { Activity contents at the time of the } \\
\text { measurement according to the phase }\end{array}$ & $\begin{array}{l}\text { U nursery } \\
\text { school }(\mathrm{n}=30)\end{array}$ & $\begin{array}{c}\text { K nursery scho } \\
(\mathrm{n}=54)\end{array}$ & $\begin{array}{l}\text { F kindergarten } \\
(\mathrm{n}=49)\end{array}$ & $\begin{array}{c}\text { Y kindergarten } \\
(\mathrm{n}=45)\end{array}$ \\
\hline $\begin{array}{l}\text { First } \\
\text { pahse }\end{array}$ & $\begin{array}{l}\text { Music play and self-introduction } \\
\text { with the song, "What is your name?" }\end{array}$ & $\begin{array}{l}\text { May } 20 \\
\text { in } 2016 \\
\text { June24 } \\
\text { in } 2016\end{array}$ & $\begin{array}{l}\text { May } 23 \\
\text { in } 2016 \\
\text { June } 20 \\
\text { in } 2016\end{array}$ & $\begin{array}{l}\text { May } 30 \\
\text { in } 2017 \\
\text { June } 02 \\
\text { in } 2017\end{array}$ & $\begin{array}{l}\text { May 26 } \\
\text { in } 2017 \\
\text { June } 23 \\
\text { in } 2017\end{array}$ \\
\hline $\begin{array}{l}\text { Second } \\
\text { phase }\end{array}$ & $\begin{array}{l}\text { Song play of "Shopping at a } \\
\text { Bakery." }\end{array}$ & $\begin{array}{l}\text { July } 15 \\
\text { in } 2016 \\
\text { August } 19 \\
\text { in } 2016\end{array}$ & $\begin{array}{l}\text { July } 11 \\
\text { in } 2016 \\
\text { August } 15 \\
\text { in } 2016\end{array}$ & $\begin{array}{l}\text { July } 04 \\
\text { in } 2017 \\
\text { July } 11 \\
\text { in } 2017\end{array}$ & $\begin{array}{l}\text { July } 14 \\
\text { in } 2017 \\
\text { September08 } \\
\text { in } 2017\end{array}$ \\
\hline $\begin{array}{l}\text { Third } \\
\text { phase }\end{array}$ & $\begin{array}{l}\text { The movement of the lion during } \\
\text { "The Grand March of the Lion". }\end{array}$ & $\begin{array}{l}\text { September } 23 \\
\text { in } 2016\end{array}$ & $\begin{array}{l}\text { September } 5 \\
\text { in } 2016 \\
\text { October } 30 \\
\text { in } 2016\end{array}$ & $\begin{array}{l}\text { October } 13 \\
\text { in } 2017\end{array}$ & $\begin{array}{l}\text { October } 20 \\
\text { in } 2017\end{array}$ \\
\hline $\begin{array}{l}\text { Fourth } \\
\text { phase }\end{array}$ & $\begin{array}{l}\text { Movement to play a musical } \\
\text { instrument while singing a song } \\
\text { including a story } \\
\text { Question and reply song }\end{array}$ & $\begin{array}{l}\text { December } 16 \\
\text { in } 2016\end{array}$ & $\begin{array}{l}\text { December } 26 \\
\text { in } 2016 \\
\text { January } 25 \\
\text { in } 2017\end{array}$ & $\begin{array}{l}\text { December05 } \\
\text { in } 2017 \\
\text { January } 16 \\
\text { in } 2018\end{array}$ & $\begin{array}{l}\text { December } 15 \\
\text { in } 2017 \\
\text { January12 } \\
\text { in } 2018\end{array}$ \\
\hline
\end{tabular}

Table1 shows the graded activities from the practical process of the MEB program to do the movement analysis through the MVN system.

\subsection{Measurement of Body Movement in Musical Expression during the Practice of MEB Program by MVN System}

The author used the latest developed MVN system as 3D motion capture to collect data at a frame rate of $60 \mathrm{~Hz}$ regarding body movement in musical expression every phase during the practice of MEB program.

The MVN system utilizes seventeen motion trackers to monitor full human body activity such as head, arm, hands and feet. Calibrated with body lengths such as arm-span, leg length, waist position, one by one 3-year-old, 4-year-old and 5-year-old child was surveyed in two nursery schools in 2016 and two kindergartens in 2017.

MVN system is a light weight and compact device which provides less constrained environment for even small sized children. MVN motion capture data include seventeen measurement points of body parts such as the pelvis, head, right shoulder, right hand, and right foot. The children's movement in musical expression were measured one by one during playing the piano accompaniment.

Figure 1 shows a 4-year-old boy wearing MVN's 17 motion trackers. Each child needed 5-10 minutes including the measurement time of 30 seconds.

Figure 2 shows an image of PC screen when 3D motion capture is executed. The body movement of the participant child is captured in real time when the MVN system is executed and shown on the PC screen. Concerning the MVN data, the moving distance, the moving average of velocity, the moving average of acceleration, and the movement smoothness (the moving average of velocity/ the moving average of acceleration) were calculated as feature quantities. The author tried to find the characteristics of element of movement in musical expression in early childhood through the analysis by age and activity phase. 


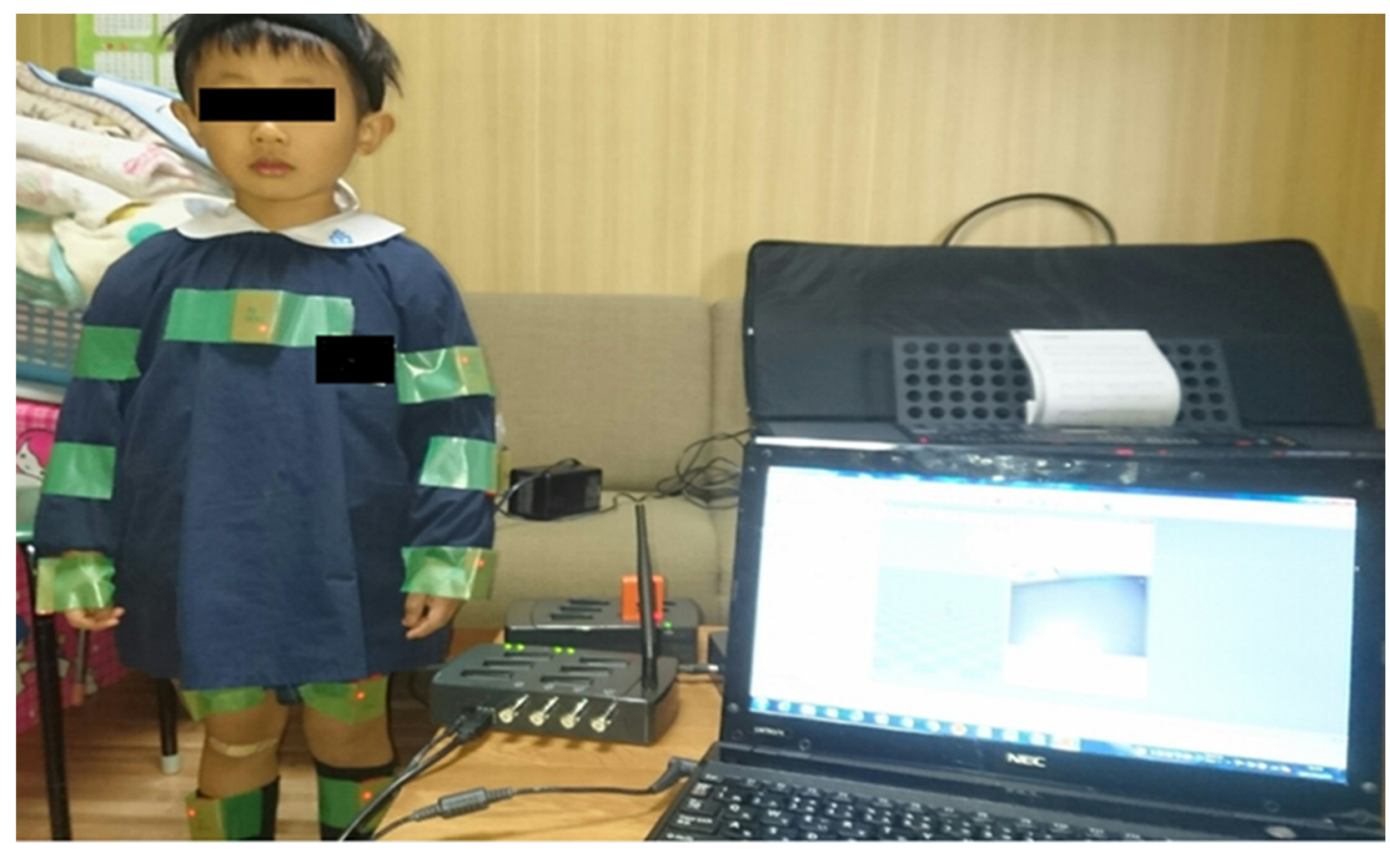

Figure 1. A 4-year-old Boy Wearing MVN's 17 Motion Trackers

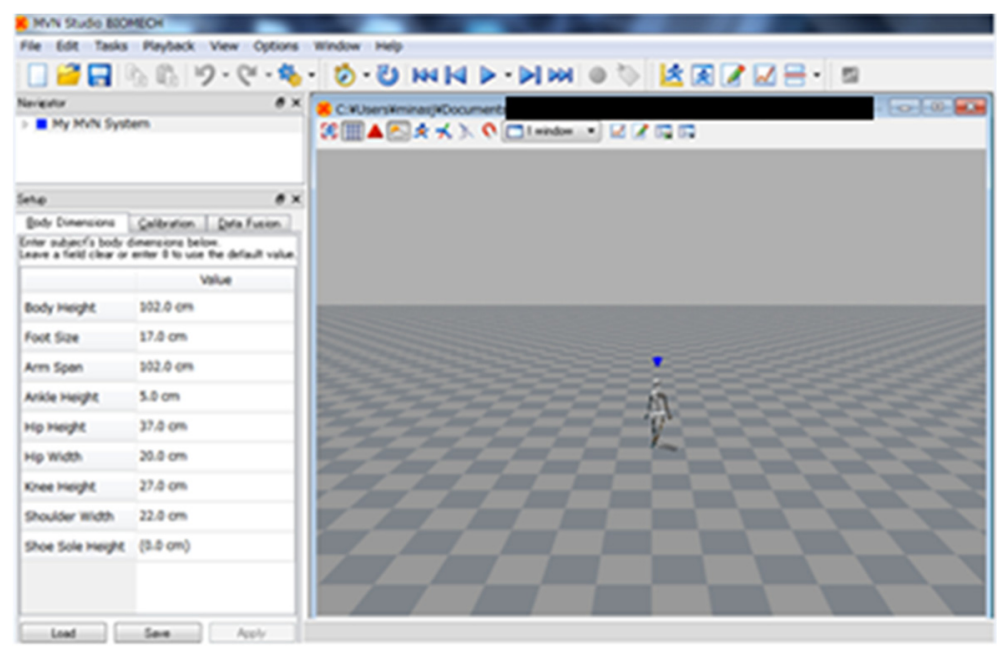

Figure 2. PC Screen when 3D Motion Capture is Executed

\subsection{Quantitative Analysis of the Motion Capture Data}

The author analyzed the measured MVN data from the first phase to the fourth phase of the MEB program, 4 facilities, and the age of the participant child (3-year-old, 4- year-old, and 5- year-old children). A three-way ANOVA (non-repeated four standards as facilities, non-repeated three standards as ages and non-repeated four standards as MEB phase) was applied in order to find statistically significant difference between relevant measures.

\subsection{Classification Model Training for Evaluation of Degree of Musical Development by Machine Learning}

The authors analyzed moving images at the time of acquiring MVN data and evaluated the musical development degree of each child at three levels: high, medium and low. (High: 15, Medium: 27, Low: 34 people.) Machine learning classification model training was conducted with the feature quantity of each child as a factor, and three level evaluation as categorical dependent variable. 


\section{Results}

Table 2. Changes in the Movement Distance of the Right hand of UKFY Facilities by Activity Phase

\begin{tabular}{|c|c|c|c|c|c|}
\hline phase & facility & age & Mean & SD & $\mathrm{N}$ \\
\hline \multirow{12}{*}{ 1st phase } & K nursery & 3 -year-old & 4.88 & 2.33 & 17 \\
\hline & school & 4-year-old & 5.03 & 2.42 & 17 \\
\hline & & 5-year-old & 5.61 & 2.19 & 20 \\
\hline & U nursery & 3-year-old & 3.83 & 1.28 & 11 \\
\hline & school & 4-year-old & 4.20 & 2.29 & 8 \\
\hline & & 5-year-old & 4.95 & 1.90 & 10 \\
\hline & F & 3 -year-old & 1.78 & 0.87 & 18 \\
\hline & kindergarten & 4-year-old & 1.73 & 1.44 & 14 \\
\hline & & 5-year-old & 1.63 & 1.60 & 15 \\
\hline & $\mathrm{Y}$ & 3 -year-old & 2.30 & 1.71 & 16 \\
\hline & kindergarten & 4-year-old & 1.81 & 1.16 & 14 \\
\hline & & 5-year-old & 3.32 & 2.40 & 15 \\
\hline \multirow{12}{*}{ 2nd phase } & K nursery & 3-year-old & 3.36 & 2.34 & 8 \\
\hline & school & 4-year-old & 4.59 & 2.06 & 14 \\
\hline & & 5-year-old & 4.51 & 1.44 & 17 \\
\hline & U nursery & 3 -year-old & 3.25 & 1.31 & 10 \\
\hline & school & 4-year-old & 3.09 & 1.21 & 9 \\
\hline & & 5-year-old & 5.11 & 2.21 & 11 \\
\hline & $\mathrm{F}$ & 3 -year-old & 3.06 & 1.20 & 13 \\
\hline & kindergarten & 4-year-old & 2.80 & 1.77 & 14 \\
\hline & & 5-year-old & 3.30 & 0.95 & 16 \\
\hline & $\mathrm{Y}$ & 3 -year-old & 3.10 & 1.63 & 15 \\
\hline & kindergarten & 4-year-old & 2.58 & 1.12 & 14 \\
\hline & & 5-year-old & 3.81 & 0.93 & 14 \\
\hline \multirow{12}{*}{ 3rd phase } & K nursery & 3-year-old & 18.43 & 8.42 & 18 \\
\hline & school & 4-year-old & 21.37 & 10.49 & 17 \\
\hline & & 5-year-old & 13.21 & 9.22 & 18 \\
\hline & U nursery & 3 -year-old & 5.13 & 2.75 & 11 \\
\hline & school & 4-year-old & 3.11 & 1.13 & 8 \\
\hline & & 5-year-old & 7.39 & 3.39 & 9 \\
\hline & $\mathrm{F}$ & 3 -year-old & 12.36 & 7.44 & 17 \\
\hline & kindergarten & 4-year-old & 8.68 & 5.56 & 12 \\
\hline & & 5-year-old & 12.36 & 7.44 & 17 \\
\hline & $\mathrm{Y}$ & 3 -year-old & 7.22 & 4.85 & 13 \\
\hline & kindergarten & 4-year-old & 8.23 & 4.90 & 13 \\
\hline & & 5-year-old & 14.20 & 5.76 & 14 \\
\hline \multirow{12}{*}{ 4th phase } & K nursery & 3-year-old & 5.11 & 2.78 & 15 \\
\hline & school & 4-year-old & 7.58 & 3.72 & 12 \\
\hline & & 5 -year-old & 6.09 & 2.86 & 19 \\
\hline & U nursery & 3 -year-old & 10.83 & 6.17 & 9 \\
\hline & school & 4-year-old & 3.61 & 2.12 & 9 \\
\hline & & 5 -year-old & 5.01 & 1.60 & 10 \\
\hline & $\mathrm{F}$ & 3 -year-old & 4.86 & 2.09 & 13 \\
\hline & kindergarten & 4-year-old & 5.03 & 3.05 & 12 \\
\hline & & 5-year-old & 5.60 & 3.78 & 16 \\
\hline & $\mathrm{Y}$ & 3 -year-old & 4.87 & 1.75 & 13 \\
\hline & kindergarten & 4-year-old & 6.14 & 2.00 & 14 \\
\hline & & 5-year-old & 7.29 & 3.47 & 15 \\
\hline
\end{tabular}


In this section, firstly, a summary of characteristic data is described by showing a part of the result analysis which is a combination of acquired data of 2016 and 2017. Secondly, the process of machine learning and the result on the extracted feature quantity are discribed.

\subsection{Characteristic Results Regarding a Three-Way Non-Repeated Analysis}

From motion capture output, 21 values regarding such as the moving distance, the moving average of velocity, the moving average of acceleration, smoothness of movement, (pelvis, head, right shoulder, right hand, right foot) and moving distance between right and left hands interval were calculated. The smoothness of movement was calculated by the ratio of moving average velocity / moving average acceleration (Burger, 2013). Concerning the result of a three-way ANOVA (non-repeated four standards as facilities, non-repeated three standards as ages and non-repeated four standards as MEB phase), the moving distance and the moving average acceleration of pelvis and the right hand were characteristic. Next, an analysis result of the right hand moving distance is shown.

\subsubsection{Change of the Moving Distance of Right Hand}

Table 2 shows the result of measurement data of the moving distance of the right hand by the activity phase/ facilities (nursery school and kindergarten)/age.

As shown in Table2, the author observed a statistically significant difference in the average moving distance of right hand in the third phase of MEB program between phase and different ages as a result of analysis concerning the moving distance of right hand.

Table 3. A Main Effect/ Interaction of the Test

\begin{tabular}{lrrrl}
\hline factor & $d f$ & \multicolumn{2}{c}{$F$} & $\begin{array}{l}\text { significance } \\
\text { probability }\end{array}$ \\
\hline phase & 3 & 117.785 & $p<.005$ \\
facility & 3 & 24.984 & $p<.005$ \\
phase * facility & 9 & 14.62 & $p<.005$ \\
facility * age & 6 & 5.579 & $p<.005$ \\
phase * facility * age & 18 & 3.457 & $p<.005$ \\
\hline
\end{tabular}

As shown in Table 3, a main effect/ interaction of the test showed a statistically significant difference shown as table3 (phase: $\mathrm{F}(3,606)=117.785, \mathrm{p}<.005)$, facility: $\mathrm{F}(3,606)=24.984, \mathrm{p}<.005$, phase * facility: $\mathrm{F}(9,606)=14.620$, $\mathrm{p}<.005$, facility * age: $\mathrm{F}(6,606)=5.579, \mathrm{p}<.005$, phase * facility * age: $\mathrm{F}(18,606)=3.457, \mathrm{p}<.005)$. The test regarding simple main effect with multiple comparisons was carried out by Bonferroni method.

Concerning the facility factor/ age factor * phase factor, the simple main effect was statistically significant in 3-yearold $(F(3,606)=47.832, p<.005)$, 4- year- old $(F(3,606)=62.538, p<.005)$ and 5-year-old $(F(3,606)=17.050, p$ $<.005)$ in K nursery school and 3-year-old $(F(3,606)=6.821, p<.005)$ in U nursery school. Significant differences regarding 3-year-old $(F(3,606)=22.808, p<.005)$, 4- year- old $(F(3,606)=7.267, p<.005)$ and 5- year- old $(F(3$, $606)=22.051, p<.005)$ in F kindergarten. Significant differences were observed in 4- year- old $(F(3,606)=7.479$, $p<.005)$, and 5- year- old $(F(3,606)=21.651, p<.005)$ in Y kindergarten. As a result of multiple comparison, 3 -year- old and 4- year- old during the third phase in K nursery school showed a significant difference. The average data of K nursery school was significantly high.

Concerning the facility factor, the simple main effect was statistically significant in 3-year-old $(F(3,606)=31.427$, $p<.005)$, 4-year-old $(F(3,606)=49.306, p<.005), 5$-year-old $(F(3,606)=5.701, p<.005)$ during the third phase. The data of 3-year-old $(F(3,606)=5.145, p<.005)$ during the fourth phase also showed a significant difference. As a result of multiple comparison, 5-year-old during the first phase in K nursery school was larger than F kindergartener's data. During the third phase, 3-year-old in K nursery school was larger than F kindergarten's data. Regarding 4-year-old, K nursery school was significantly larger than F kindergarten, F \&Y kindergarten's data were larger than U nursery school's data. U nursery school was significantly small regarding 5-year-old, and 3-year-old in U nursery school was large during the fourth phase.

Concerning age factor, the simple main effect was statistically significant in the third phase (K nursery school: $F(2$, $606)=18.215, p<.005$, Y kindergarten: $F(2,606)=11.740, p<.005)$, and the fourth phase ( U nursery school: $F(2$, 
$606)=8.036, p<.005$ ). As a result of multiple comparison, 3-year-old and 4-year-old in K nursery school, 3-year-old, 5-year-old in F kindergarten, and 5-year-old in Y kindergarten during the third phase. 3-year-old in U nursery school showed large data during the fourth phase. Figure 2 shows the change of moving distance regarding right hand of 5 -year-old by facility by phase of MEB program. The moving distance of right hand was the largest in all calculated data during the third phase.

\subsubsection{Change of the Moving Average of Acceleration of Pelvis}

As a result of a three way non-repeated ANOVA regarding the moving average of acceleration of pelvis as well as the moving distance of right hand, a main effect/ interaction of the test showed a statistically significant difference (phase: $F(3,606)=186.412, p<.005$, facility: $F(3,606)=10.019, p<.005$, age: $F(2,606)=5.829, p<.005$, phase* facility: $F(9,606)=3.727, p<.005$, phase* age: $F(6,606)=5.595, p<.005)$.

Concerning the facility factor/ phase* facility* age, the simple main effect was statistically significant ( $\mathrm{K}$ nursery school: 3 -year-old $(F(3,606)=42.845, p<.005), 4$-year-old $(F(3,606)=29.123, p<.005), 5$-year-old $(F(3,606)=27.025$, $p<.005)$, U nursery school: 3-year-old $(F(3,606)=5.049, p<.005), 5$-year-old $(F(3,606)=24.692, p<.005)$. As a result of multiple comparison, large data were observed in the all ages in K nursery school, F \& Y kindergartens, 3-year-old and 5-year-old in U nursery school during the third phase.

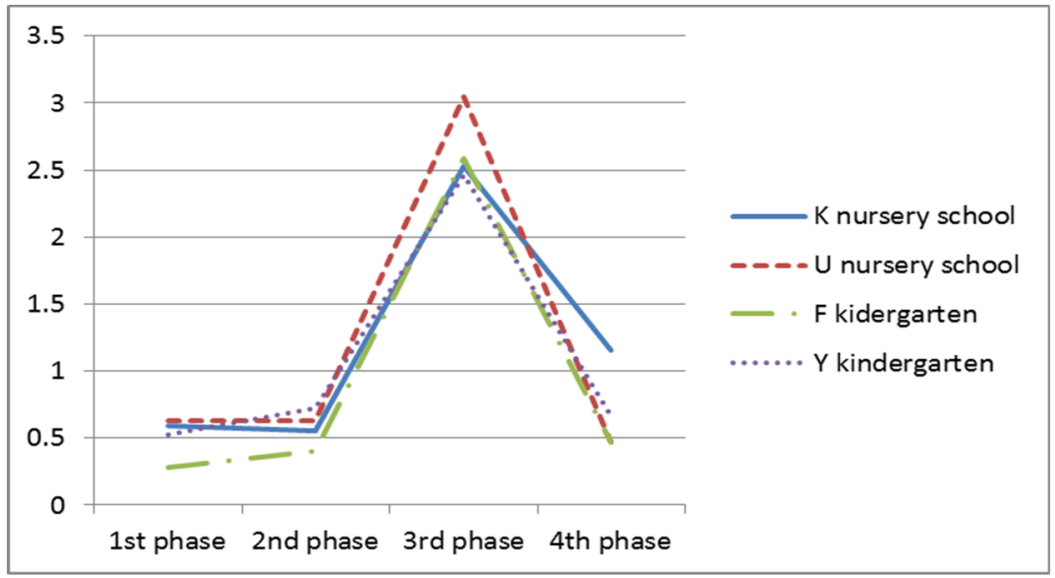

Figure 3. Change of Moving Average of Acceleration Regarding Pelvis of 5-year-old by Facility by Phase of MEB Program $\left(\mathrm{m} / \mathrm{s}^{2}\right)$

Figure 3 shows change of moving average of acceleration regarding pelvis of 5-year-old by facility by phase of MEB program. 5-year-old children in four facilities showed similar changes by activity phase and large value at the third phase including activities to recognize musical elements in MEB program.

\subsubsection{Change of the Movement Smoothness of Right Foot}

As a result of a three way, non-repeated ANOVA regarding the moving average of acceleration of pelvis as well as the moving distance of right hand, a main effect/ interaction of the test showed a statistically significant difference (phase: $F(3,606)=5.175, p<.005$, facility: $F(3,606)=75.344, p<.005)$.

Concerning the facility factor/ phase* facility* age, the simple main effect was statistically significant (K nursery school: 3 -year-old $(F(3,606)=3.821, p<.005)$, 4-year-old $(F(3,606)=6.571, p<.005), 5$-year-old $(F(3,606)=6.525$, $p<.005)$, U nursery school: 5 -year-old $(F(3,606)=7.138, p<.005))$. As a result of multiple comparison, the data of the fourth phase was larger than the third phase regarding 3-year-old children, the data of the second and the fourth phase were larger than the third phase regarding 4-year-old children, and the data of the fourth phase was larger than the first phase regarding 5-year-old children in K nursery school. The data of the second phase was larger than the third phase regarding 4-year-old and 5-year-old children in U nursery school.

Concerning the facility factor, the simple main effect was statistically significant (3-year-old in the first phase: $F(3$, $606)=6.555, p<.005$, the second phase: 3 -year-old $(F(3,606)=9.169, p<.005), 4$-year-old $(F(3,606)=15.142, p<.005)$, 5 -year-old $(F(3,606)=16.963, p<.005)$, the fourth phase: 3-year-old $(F(3,606)=8.480, p<.005)$, 4-year-old $(F(3$, $606)=11.647, p<.005), 5$-year-old $(F(3,606)=17.637, p<.005))$. As a result of multiple comparison, the data of $\mathrm{U}$ nursery school was larger than F \& Y kindergartens regarding 3-year-old children, and the data of K nursery school 
was larger than $\mathrm{F}$ kindergarten regarding 4-year-old children in the first phase. The data of $\mathrm{K} \& \mathrm{U}$ nursery schools were larger than F \& Y kindergartens regarding 3-year-old, 4-year-old and 5-year-old children in the second phase, and the data of $\mathrm{K}$ nursery school was larger than $\mathrm{F} \& \mathrm{Y}$ kindergartens regarding 5-year-old children in the third phase. The data of K nursery school was larger than F \& Y kindergartens regarding 3-year-old, 4-year-old and 5-year-old children in the fourth phase. Concerning age factor, none of the simple main effects were significant, and there was no significant difference in the results of multiple comparisons.

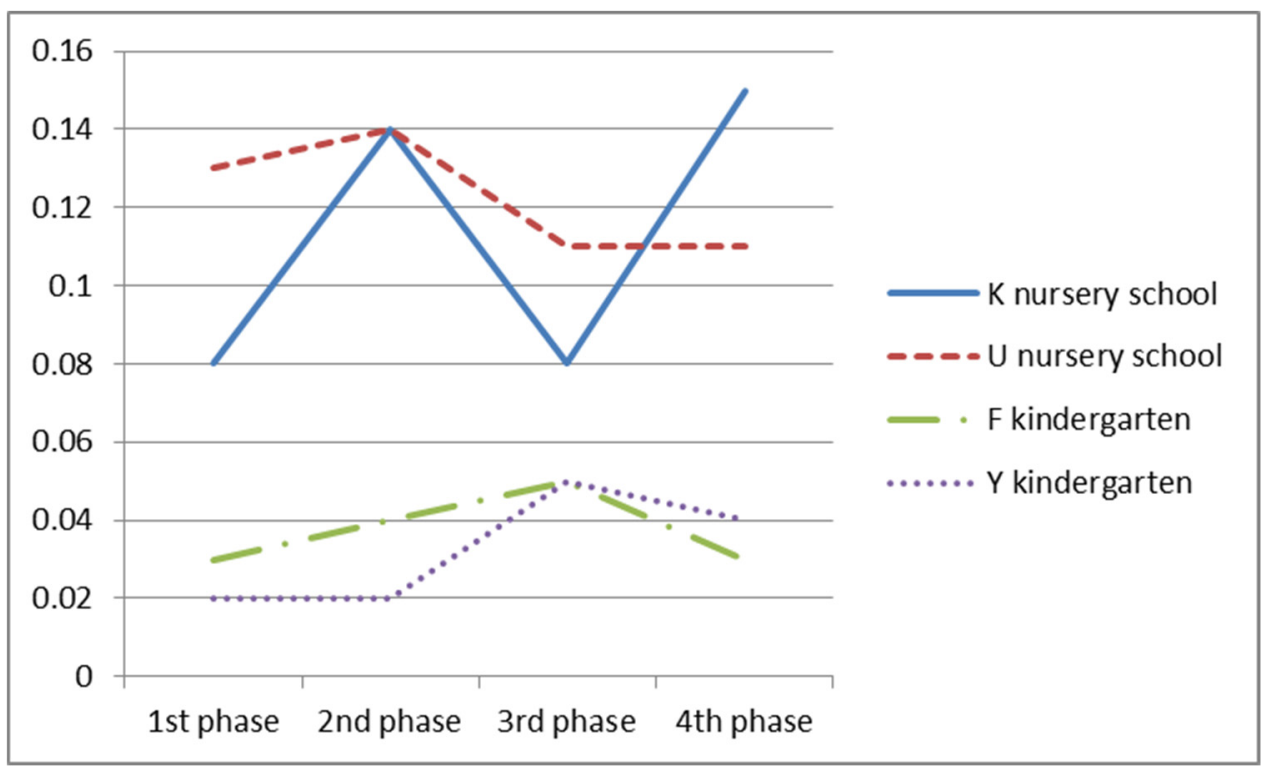

Figure 4. Change of the Movement Smoothness of Right Foot Regarding 3-year-old by Facility by Phase of MEB Program (m)

After all, the change of data of $\mathrm{K} \& \mathrm{U}$ nursery schools was more remarkable than $\mathrm{F} \& \mathrm{Y}$ kindergartens regarding the movement smoothness of right foot shown as Figure 4.

In this way, based on the result of a three way, non-repeated ANOVA on all MVN measurement data, the author tried model classification and discrimination of feature quantities by machine learning.

4.2 Method of Evaluation Support by Machine Learning When Evaluating the Musical Development Level of Early Childhood Children

\subsubsection{Attempt of Machine Learning}

In recent studies, machine learning is used for behavioral learning support (Matsumoto, Mikami, Kawamura, \& Kojima, 2014) such as action recognition and personal recognition method in everyday life (Kodama, Oba, \& Ishii, 2015; Takada, Kitasuka, and Aritsugi, 2012) and elementary school students' athletic back hip circle (Matsumoto, Mikami, Kawamura, \& Kojima, 2014). Studies regarding ICT also show technology product as ICT tool such as computer (Hall \& Higgins, 2002) and robotics in an early years classroom (McDonald \& Howell, 2012). Although no research report with machine learning on musical expressions has been seen yet, the author has examined the possibility and practicality of the method as follows.

Early childhood educators usually evaluate the musical development of young children from the body movement in musical expression and educate them to develop balanced evolution regarding musical elements such as sound strength, duration of sound, pitch, and rhythm based on their own experience. Evaluation of musical development degree has no clear scoring rule including a specific action such as Japanese traditional dance, but experienced child educators generally come into line regarding achieved level of children's expression. Teachers who involved in the MVN measurement in the four facilities tended to show similar evaluation. Each 3 early childhood teachers at $\mathrm{K} \& \mathrm{U}$ nursery school and 1 early childhood teacher each at F \& Y kindergarten were involved in the evaluation of children's musical expression with naked eyes at the time of MVN measurement.

If machine learning can fairly handle data captured by MVN, certain level of objective and transparent evaluation can be shared easily, though, such precision is not so high as that of experienced and skillful educators.

If this evaluation support system is feasible, it could be used by educators with limited experience. Machine based 
evaluation can be conducted even when there is no early childhood educator, so it can be frequently used to evaluate activities of musical expression and it is useful for early childhood education.

As machine learning herewith, J48 of decision tree and Random Tree, Sequential Minimal Optimization algorithm (SMO), Support Vector Machine (SVM), Multilayer Perceptron of Neural Network were used as classifiers in this article. Using the MVN system, data was acquired from 3-year- old, 4- year- old, and 5- year-old children $(\mathrm{n}=76)$ of U \& K nursery schools in December 2016 and January 2017, and at the same time, in conjunction with movement of each child was recorded. After calculating 13 kinds of feature kinetic quantities (moving distance/ moving average of velocity/ moving average of acceleration of pelvis/ right hand/ right foot, the movement smoothness, and left and right hand spacing), the author evaluated the musical development level of each child based on recorded video image by three levels of high/ medium/ low (high: $n=15$, medium: $n=27$, low: $n=34$ ). Classification model training was carried out using this feature quantity (factor) and three level evaluation (categorical dependent variable) as training objects of machine learning.

As a result of machine learning, the classification accuracy was $47.3 \%$ for J48, $42.1 \%$ for RandomTree and $46.1 \%$ for SMO.

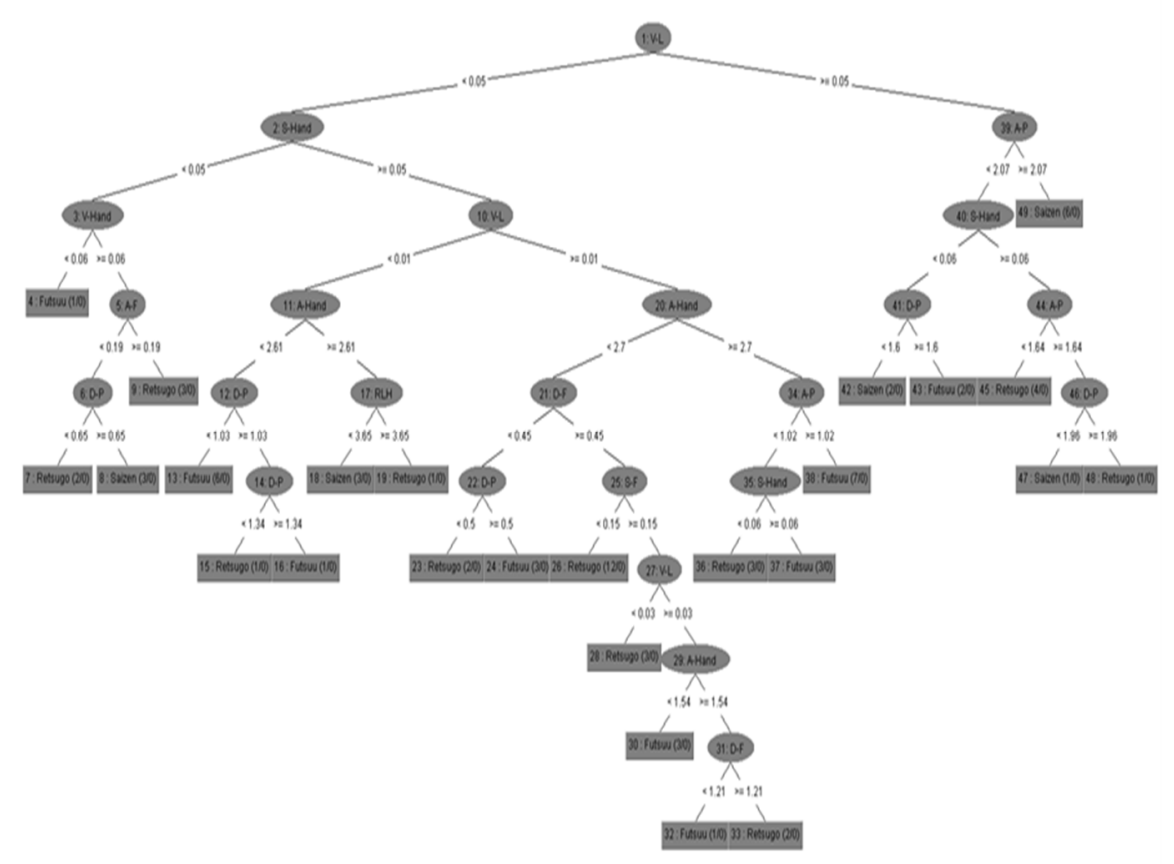

Figure 5. The Decision Tree of RandomTree

Figure 5 shows the decision tree of RandomTree. 
The Multilayer Perceptron Neural Networks

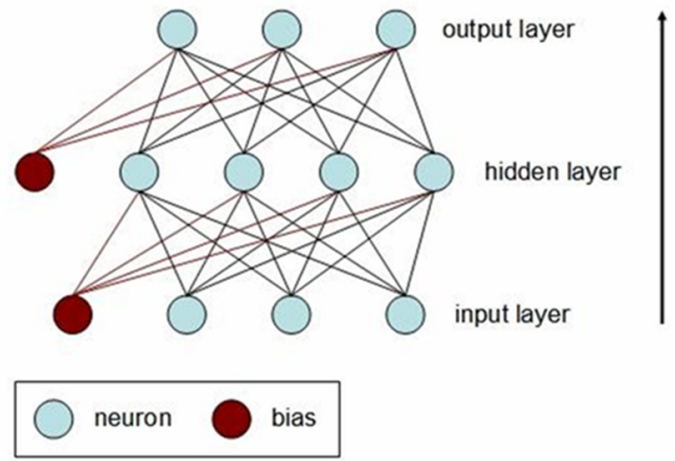

Figure 6. Schematic Diagram of Multilayer Perceptron (STATISTICA)

The classification accuracy of multilayer perceptron was $63.6 \%$ ( $70 \%$ for learning sample, $30 \%$ for test sample). Multilayer perceptron diagram is described like as Figure 6.

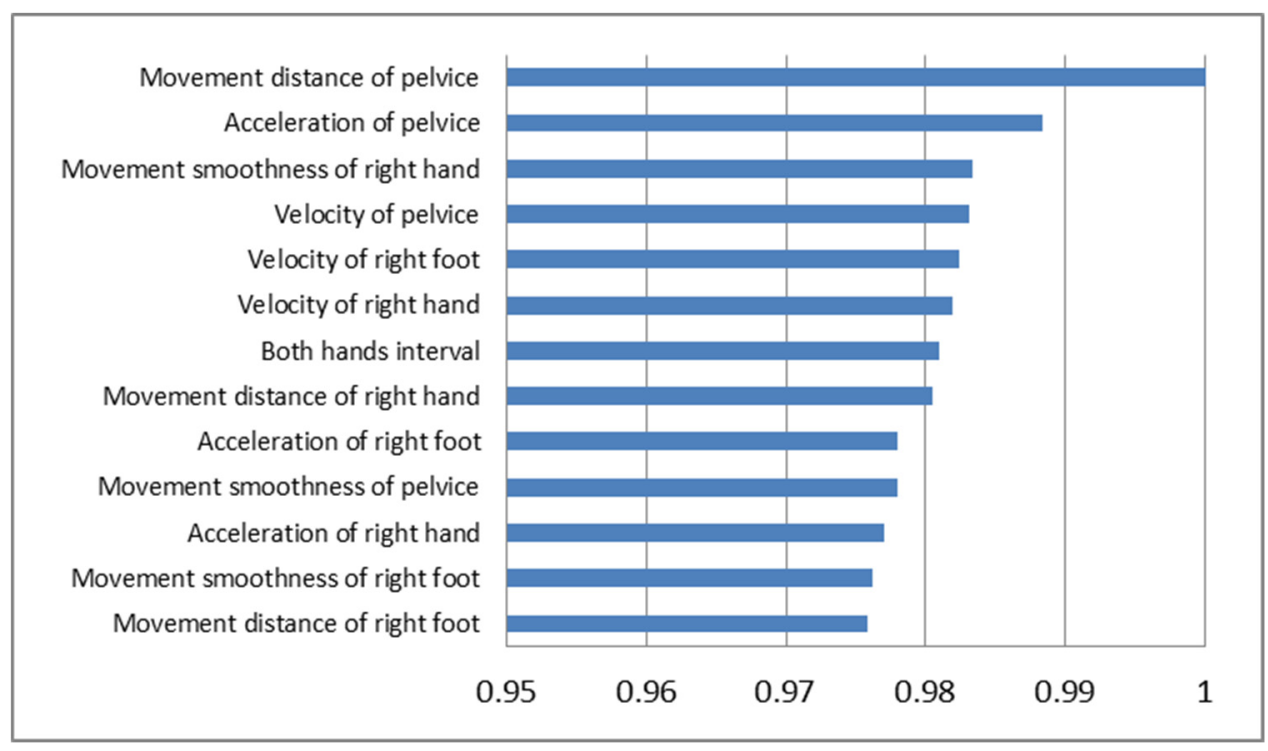

Figure 7. Normalized Sensitivity of Feature Quantity

The sensitivity of the feature quantity to the musical development level of the multilayer perceptron was as shown in Figure 7. As Figure 7 showed, high sensitivities were observed in the moving distance, acceleration and velocity regarding pelvis, movement smoothness of right hand, and the moving average velocity of right foot.

\subsubsection{Training of Machine Learning}

The author now acquired prameter sets of variables as learning results of 76 children. As multilayer perceptron showed highest classification accuracy in learning process, the author utilized multilayer perceptoron on different group of children. As a different group, using feature quantities of 3-year- old, 4- year- old, and 5- year- old children $(\mathrm{n}=83)$ in $\mathrm{F} \& \mathrm{Y}$ kindergartens where 3D data capturing and simultaneous video recording were obtained in December 2017 and January 2018, conducting acquired variables learned from 76 children, categorical dependent variables were calculated in multilayer perceptron. The author evaluated the musical development of each child from the video recordings of 83 children (high: 31, medium: 28, low: 24) with three grades of high, middle and low, and compared with calculation results. Using the model variables derived from the training of machine learning (76 children), the musical development level of body movements $(n=83)$ was calculated and compared with the results judged from the recorded video image, the following confusion matrix was acquired. Classification accuracy was $32.6 \%$. 
Table 4. Result of Classification

\begin{tabular}{lllll}
\hline & & \multicolumn{3}{c}{ Prediction } \\
\cline { 3 - 5 } & & High & Medium & Low \\
\hline \multirow{3}{*}{ Actual } & High & 1 & 11 & 19 \\
& Medium & 0 & 5 & 23 \\
& Low & 0 & 2 & 22 \\
\hline
\end{tabular}

As shown in Table 4, the classification accuracy applying trained parameters on different group was lower than the accuracy in training, but the prediction deviation was consistent, and it was lower than the degree of development determined from the recorded video image.

\section{Discussion}

In this article, in order to extract the developmental characteristics of musical expressions in early childhood from a viewpoint of change regarding elements of body movement and to generate an evaluation model based on those feature quantities, the author presented a method of model classification.

\subsection{Characteristics of Body Movement in Musical Expression}

Musical development degree in early childhood was tried to evaluate using the data regarding 3-year-old, 4-year-old, and 5-year-old children acquired by 3D motion capture in two nursery schools in 2016 and two kindergartens in 2017. As a result of a three way, non-repeated ANOVA, a significant difference was statistically observed in the movement of right hand such as the moving distance of right hand shown as result1.

Characteristic results regarding a three-way non-repeated analysis showed that change of the moving distance and the moving average acceleration of pelvis and the right hand were remarkable during the third phase of MEB program. The third phase's activities include various musical experiences integrated musical expression with dramatization using rhythmic pattern with reply song to encourage the recognition of musical elements in early childhood. The result of movement smoothness regarding right foot showed that the data of children in $\mathrm{K} \& \mathrm{U}$ nursery schools were significantly larger than the data of children in F \& Y kindergartens. 3-year-old, 4-year-old, and 5 -year-old children can belong to nursery school or kindergarten in Japan. The time spent by children is longer in a nursery school than in a kindergarten. Children in nursery school can also have time to fully experience so that children reproduce the activity content of musical expression in a play. Therefore, the children in $\mathrm{K} \& \mathrm{U}$ nursery schools seem to have shown movement smoothness more than children in F \& Y kindergartens. Furthermore, above results regarding the moving average of distance and the moving average of acceleration in a childcare form following Montessori method such as K nursery school and $\mathrm{Y}$ kindergarten generally tended to present larger value than a play-centered childcare form in U nursery school and $\mathrm{F}$ kindergarten.

\subsection{Method of Evaluation Support by Machine Learning}

The author tried to report here an application of machine learning to evaluate achievement level of musical expression of body movement. The idea of exploiting 3D motion capture data for machine learning came in mind when the author was processing ANOVA and observed soft patterns of statistical results. Given the body parts based kinetics data, it is suspected varieties of classifiers can be tested and compared. The author chose several classifiers, but among them, and expected multilayer perceptron might show fair results due to its robustness as well as SVM or Random Tree might perform well on widely varying data sets (Fernandez-Delgado, et al,. 2014). The result was in favor of multilayer perceptron, suggesting its high accuracy and flexible setting of model nodes or layers. SVM or other models were weaker than multilayer perceptron. The author found it interesting that multilayer perceptron evaluation results showed less awkward output than other classifiers. For example, for randomly picked up results cases, video image was reviewed for evaluator eyeballs and evaluation results of models were compared. Multilayer perceptron results generally agreed with evaluator eyeballs but other models showed some low level cases as high level case.

When the evaluation of the musical development degree acquired from the recorded video image is modeled by machine learning using the feature quantity of the motion capture data, a strong relationship between pelvic movement and musical development degree has been shown. As a result of machine learning, the classification accuracy of multilayer perceptron was recognized. Based on the sensitivity of the feature quantity to the musical 
development level during training of the multilayer perceptron, the pelvic movement supporting the center of gravity of the body was thought to be strongly related to musical development. Therefore, instruction and support for pelvic movements was considered to be one of the important points of focus for the development of body movement in musical expression in early childhood.

\section{Conclusion}

This study tried to analyze characteristics of children's body movements utilizing 3D motion capture device. The analysis was mainly two fold, based on ANOVA and based on machine learning. ANOVA was applied on multi stage of developmental stage transitions incorporating age difference variable as well as influence caused by educational methodological difference. In comparison to ANOVA, machine learning classifier model, especially multilayer perceptron, was applied on a group of children with learning variables obtained from another group in order to evaluate respective maturity of musical expression in body movement as a snapshot.

The empirical findings of ANOVA yield a range of insights into evolution of body movement in quantitative manner. Some of statistically significant kinetics of specific body parts give focus points on evolution manifestation.

Also, machine learning results hightlight usefulness of multilayer perceptron as support tool for educators who have not yet accumulated evaluation experience of musical expression of body movement.

Emprical results of this article with quantitative analysis will have implications for educators who are exploring music expression from multiple aspects.

Regarding the evaluation of the musical development level by the multilayer perceptron, in order to improve the classification accuracy, methods such as review of feature quantity, increase of sample data, deep learning and the like can be considered. Using evaluation results of multiple early childhood educators for model training, it may be possible to reduce the deviation of evaluation among experienced early childhood educators.

In the future, by improving the prediction accuracy of this method, it is considered that it is possible to predict the musical development degree from the motion capture data with high accuracy even for early childhood educators with little experience, and to conduct effective music education in early childhood.

\section{Acknowledgements}

This work was supported by JSPS KAKENHI Grant Number 16K04579.

\section{References}

Ballema, G. (2005). Year' round dramatic pla. Michigan, Frank Schaffer Publications.

Burger, B. (2013). Move the way you feel: Effects of musical features, perceived emotions, and personality on music-induced movement, $\mathrm{PhD}$ thesis, Department of Music, University of Jyväskylä, Finland.

Burger, B., Thompson, M., Saarikallio, S., Luck, G., \& Toiviainen, P. (2013). Influences of rhythm- and timbre-related musical features on characteristics of music-induced movement. Frontiers in Psychology, 4(183), 1-9. https://doi.org/ 10.3389/fpsyg.2013.00183

Custodero, L. (1999). Construction of musical understandings: The cognitive- flow interface, Cognitive processes of children engaged in musical activity Conference, 25p, files.eric.ed.gov/fulltext/ED460061.pdf

Dahl, S., \& Friberg, A. (2007). Visual perception of expressiveness in musicians' body movements. Music Perception, 24, 433-454. https://doi.org/ 10.1525/mp.2007.24.5.433

Fernandez-Delgado, M., Cernadas, E., Barro, S., \& Amorim, D. (2014). Do we need hundreds of classifiers to solve real world classification problems? Journal of Machine Learning Research, 15, 3133-3181.

Hall, E., \& Higgins, S. (2002). Embedding computer technology in developmentally appropriate practice: engaging with early years professionals' beliefs and values. Information Technology in Childhood Education, 1, 301-320.

Hannon, E., \& Johnson, S. (2005). Infants use meter to categorize rhythms and melodies: Implications for musical structure learning. Cognitive Psychology, 50, 354-377. https://doi.org/10.1016/j.cogpsych.2004.09.003

Kodama, Y., Oba, S., \& Ishii, S. (2015). Recognition of daily-life actions by machine learning methods. IEICE technical report. IBISML, 114(502), 73-78

Matsumoto, A., Mikami, H., Kawamura, H., \& Kojima, A. (2014). Support for motor learning by clustering of sports 
forms: Examine effective image features as a target children back hip circle. ITE technical report, 38, 51(0), 9-12. https://doi.org/10.11485/itetr.38.51.0_9

McDonald, S., \& Howell, J. (2012). Watching, creating and achieving: Creative technologies as a conduit for learning in the early years. British Journal of Educational Technology, 43(4), 641-651. https://doi.org/10.1111/j.1467-8535.2011.01231.x

Phiilips- Silver \& Trainor, L. (2005). Feeling the beat: Movement influences infant rhythm perception. Science, 308, 1430. https://doi.org/10.1126/science.1110922

Prentzas, J. (2013). Artificial Intelligence Methods in Early Childhood Education, Democritus University of Thrace. https://doi.org/10.1007/978-3-642-29694-9_8

Rubin, J., \& Merrion, M. (1996). Drama and Music Methods. Portsmouth: Linner Professional Publications.

Sano, M. (2013). Quantitative analysis about the educational effect of the music expression program. The 9th Asia-Pacific Symposium on Music Education Research, full-paper, 39, 1-7.

Sano, M. (2014). Contents of the music test concerning the recognition of musical elements in early childhood. Research bulletin of Osaka Shoin Women's University, 4, 67-74.

Sano, M. (2015). Characteristics of musical expressions showing the formation process of beat perception in early childhood: Through the practice of Musical Expression Bringing-up program for 5-year-old children in $\mathrm{K}$ nursery school. Japanese Journal of Music Education Practice, 12(2), 120-131. https://doi.org/10.20614/jjomep.12.2_120

Sano, M. (2016). Quantitative analysis of body movement in musical expression among three nursery schools in the different childcare forms utilizing 3D motion capture, Information and Communication Technologies in the Music Field (ICTMF), Media Musica, Central and Eastern European Online Library, 7(2), 7-18.

Sano, M. (2017). Elements of body movements in musical expression of early childhood children using 3D motion capture and evaluation support of musical development by machine learning. Japanese Journal of Educational Technology, 41 Suppl., 5-8. https://doi.org/10.15077/jjet.S41003

Sano, M. (2018). Development of a quantitative methodology to analyze the growth of recognition of musical elements in early childhood from a viewpoint of change of body movement. Asia-Pacific Journal of Research in Early Childhood Education, 12(1), 61-80. https://doi.org/10.17206/apjrece.2018.12.1.61

Sato, K., Kaiga, T., \& Watabe, S. (2010). The utilization of motion capture to support proficiency of the dancing. Japan Journal of educational technology, 34, 133-136. https://doi.org/10.15077/jjet.KJ00007086695

Takada, K., Kitasuka, T., \& Aritsugi, M. (2012). A consideration of an individual identification method by gait using a markerless motion capture device. IPSJ SIG Technical Report, 2012-EC-25, 9, 1-7.

Thelen, E. (1979). Rhythmical stereotypes in normal human infants. Anim. Behav., 27, 699-715. https://doi.org/10.1016/0003-3472(79)90006-X

Thompson, M., \& Luck, G. (2012). Exploring relationships between pianists' body movements, their expressive intentions, and structural elements of the music. Musicae Scientiae, 16, 19-40. https://doi.org/10.1177/1029864911423457

Winston, J., \& Tandy, M. (2005). Beginning Drama 4-11. London: David Fulton Publishers.

Zentner, M., \& Eerola, T. (2010) Rhythmic engagement with music in infancy, PNA, 107(3), 5768-5773. https://doi.org/10.1073/pnas.1000121107 